\title{
Comparison of efficacy, complications and TGF- 32 expression between DHS and PFNA in elderly patients with osteoporotic femoral intertrochanteric fracture
}

\author{
RAO XU*, JIANGYING RU*, FENG JI, JIE LIU, YONG JI, ZHIQUAN WU and DAI SHI \\ Department of Orthopaedics, The Affiliated Hospital of Yangzhou University, Yangzhou, Jiangsu 225000, P.R. China
}

Received November 21, 2017; Accepted March 16, 2018

DOI: $10.3892 /$ etm.2018.6177

\begin{abstract}
The aim of the present study was to compare the efficacy and complications of two fixation techniques, namely dynamic hip screw (DHS) and proximal femoral nail antirotation (PFNA), in the treatment of osteoporotic femoral intertrochanteric fracture in elderly patients, and to detect changes in transforming growth factor $\beta 2$ (TGF- $\beta 2$ ) expression in the two groups. A total of 100 elderly patients with femoral intertrochanteric fracture were randomly divided into two groups that were treated with either DHS or PFNA. Peri-operative complications were observed in the patients and ELISA was used to detect TGF- $\beta 2$ expression levels at 1, 7, 15 and 30 days after surgical treatment. The clinical efficacy and the incidence rate of complications at 3 months after the operation were compared. In comparison with the DHS group, the PFNA group had a shorter operation time, a lower bleeding volume and a shorter post-operative weight-bearing time. The contents of TGF- $\beta 2$ in the two groups at 7 days after the operation were higher than those at 1 day, reached a peak at 15 days and had gradually decreased again at 30 days after the operation. The contents of TGF- $\beta 2$ at 1,7 and 15 days in the PFNA group were higher than those at the identical time-points in the DHS group $(\mathrm{P}<0.01)$. Regarding the clinical efficacy in the two groups at 3 months of post-surgery, the rate of excellent/good efficacy in the PFNA fixation group (90.0\%) was higher than that in the DHS fixation group (74.0\%). Of note, PFNA fixation had a higher clinical efficacy, a shorter operation time, less intra-operative trauma, a relatively faster fracture healing process and fewer complications in comparison with DHS fixation, and is therefore more suitable for treating osteoporotic
\end{abstract}

Correspondence to: Mr. Jiangying $\mathrm{Ru}$, Department of Orthopaedics, The Affiliated Hospital of Yangzhou University, 368 Hanjiang Middle Road, Yangzhou, Jiangsu 225000, P.R. China E-mail: rujiangying1@126.com

*Contributed equally

Key words: dynamic hip screw, proximal femoral nail antirotation, femoral intertrochanteric fracture in the elderly, efficacy, transforming growth factor- $\beta 2$ femoral intertrochanteric fracture in the elderly. PFNA fixation is superior to DHS fixation, which may be associated with the higher level of TGF- $\beta 2$ expression in comparison with that in the DHS group.

\section{Introduction}

Femoral intertrochanteric fracture mostly occurs in the elderly aged $>60$ years, who usually have confounding factors including osteoporosis or other internal medical conditions. Conservative treatment used in clinical practice easily results in serious complications, including bedsore, urinary system infection, pulmonary infection and joint contracture due to long-term bed rest. The associated mortality rate is up to $34.6 \%$ (1). Surgical fixation is the first-line strategy for the treatment of femoral intertrochanteric fracture, as it significantly reduces the duration of bed rest, is conducive to early functional exercise and effective in avoiding complications (2,3). Methods commonly used for clinical fixation include proximal femoral nail antirotation (PFNA), PFN and dynamic hip screw (DHS) (4). PFNA is advantageous as it utilizes a small incision and produces less bleeding. However, the operation for PFNA is more difficult to perform and more expensive than DHS (4). DHS is an easy procedure that can increase pressure by sliding; however, surgical exposure is large and the incidence of coxa varus is high following surgery (4). Transforming growth factor $\beta 2$ (TGF- $\beta 2$ ) is one of the most abundant cytokines in bone tissues. It has been demonstrated that TGF- $\beta 2$ may be expressed in all stages of fracture healing, promoting the internal and cartilaginous osteogenesis during the healing process (5). In the present study, changes in TGF- $\beta 2$ expression were detected after treatment of femoral intertrochanteric fracture with DHS and PFNA fixation. The curative effects and complications of DHS and PFNA fixation were compared to explore effects of two treatment methods on the healing of femoral intertrochanteric fracture, providing a practical basis for the choice of surgical method in clinical practice.

\section{Patients and methods}

Clinical data. A total of 100 elderly patients with femoral intertrochanteric fracture (45 males and 55 females; age, 65-91 years) admitted to and treated at the Orthopedics 
Department of the Affiliated Hospital of Yangzhou University (Jiangsu, China) from January 2016 to January 2017 were included in the present study. All patients were diagnosed via pelvic anteroposterior X-ray examination, as well as anteroposterior and lateral X-ray examination of the affected hip. The inclusion criteria were as follows: Age, $\geq 65$ years; femoral neck bone density determination T-score (bone density score) $<-2.5$ standard deviations (4); and primary femoral intertrochanteric fracture. The exclusion criteria were as follows: Cortical pulverization, femoral head necrosis, Evans type II fracture or other serious complications detected in imaging eaminations, or surgical contraindications. These elderly patients were randomly divided into two groups that received DHS and PFNA treatment, respectively. Differences in age, gender and severity of the disease were not statistically significant between the two groups. The present study was approved by the ethics committee of the Affiliated Hospital of Yangzhou University (Yangzhou, China). Written informed consent was obtained from the patients and/or their guardians.

Grouping and treatment. The two groups were treated with DHS and PFNA surgical therapy, respectively. Pre-operative examinations were performed and antibiotics were routinely administered to prevent infection prior to the operation. After the operation, preventive anti-inflammatory therapy was performed, subcutaneous injection of low-molecular-weight heparin calcium was given to prevent venous thrombosis, and anti-osteoporosis drugs were administered. Drainage tubes were removed within 24-36 h based on the individual circumstances. On the day after the operation, lower limb muscle contraction exercise was commenced depending on the condition of each patient, and subsequently, joint function exercise was performed gradually. At 1, 4, 8 and 12 weeks after the operation, the time of weight-bearing ambulation was determined according to the results of X-ray examinations (Multix Select DR; Siemens, Erlangen, Germany). Patients were followed up for 3 months after discharge and had re-examinations on the affected limb joints via X-ray every month.

Observation indexes. The following operation-associated conditions of patients were observed: Operation time, intra-operative bleeding volume, post-operative weight-bearing time and length of hospital stay. The post-operative callusing time and swelling reduction time of patients were noted. Blood sampling was performed at 1,7, 15 and 30 days after the operation for TGF- $\beta 2$ determination. Fasting venous blood $(3 \mathrm{ml})$ was collected early in the morning, centrifuged for serum separation after standing for $0.5 \mathrm{~h}$ (Microfuge 16; Beckman Coulter, Brea, CA, USA) and then stored at $-70^{\circ} \mathrm{C}$. TGF- $\beta 2$ levels were determined using an ELISA kit (cat. no. 1217; Shanghai Yubo Biotechnology Co., Ltd., Shanghai, China;). A microplate reader (Synergy H1; BioTek, Winoosky, VT, USA) was adopted to measure the TGF- $\beta 2$ content.

The post-operative hip function Harris score was used as the scoring standard for efficacy (6). The efficacy score was evaluated as follows: $\geq 90$ points, excellent; $80-89$ points, good; $70-89$ points, fair; $\leq 70$ points, poor. Post-operative complications were compared between the two groups.
Statistical analysis. SPSS version 19.0 (IBM Corp., Armonk, NY, USA) was used for data processing. Values are expressed as the mean \pm standard deviation. The Student's t-test was applied for comparison between two groups. The $\chi^{2}$ test was employed for comparison among enumeration data. $\mathrm{P}<0.05$ was considered to indicate a statistically significant difference.

\section{Results}

Comparison of general clinical characteristics. Between the DHS and the PFNA group, no statistically significant differences in age, amount of hemoglobin prior to the operation, gender distribution, fracture type and complications were observed ( $\mathrm{P}>0.05$; Table I).

Comparison of peri-operative parameters between the DHS and PFNA groups. Compared with those in the DHS group, the operation time and bleeding volume were reduced, and the length of hospital stay and post-operative weight-bearing time were shortened in the PFNA group $(\mathrm{P}<0.05$ or $\mathrm{P}<0.01$; Table II).

Callusing time and swelling reduction time in DHS and PFNA group. Comparison of the recovery process in the two groups of patients indicated that the callusing time and swelling reduction time in the PFNA vs. the DHS group were 31.5 \pm 3.1 vs. $43.3 \pm 3.5$ days and $8.3 \pm 4.9$ vs. $13.6 \pm 5.0$ days, respectively, and differences were statistically significant $(\mathrm{P}<0.05)$, indicating that the PFNA group had a faster post-operative fracture healing process (Table III).

Comparison of TGF- $\beta 2$ expression between the DHS and PFNA groups. The results of the dynamic TGF- $\beta 2$ detection revealed that after the operative treatment, the TGF- $\beta 2$ content at 7 days was increased compared with that at 1 day, reached the peak at 15 days and had declined again at 30 days with the gradual healing of the fracture. The content of TGF- $\beta 2$ at $1,7,15$ and 30 days in the PFNA group was obviously higher than that at the identical time-points in the DHS group, and differences were statistically significant (Table IV).

Comparison of hip function scores between the DHS and PFNA groups. Comparison of hip function scores at 3 months after the operation indicated that in the DHS fixation group, the efficacy was rated as excellent in 38 patients, good in 6 patients, fair in 3 patients and poor in another 3 patients, with a rate of excellent and good efficacy of $74.0 \%$. In the PFNA fixation group, the efficacy was rated as excellent in 45 cases, good in 2, fair in 2 and poor in 1 patient, with a rate of excellent and good efficacy of $90.0 \%$. Statistical analysis indicated that the rate of excellent and good efficacy in the PFNA group was significantly higher than that in the DHS fixation group $(\mathrm{P}<0.05$; Table V).

Comparison of common complications between DHS and PFNA groups. In the DHS fixation group, complications occurred in 8 patients (incidence rate, 16\%), including hip varus in 2 patients, femoral shaft fracture in 2 patients, cutout of femoral head in 1 patient, fracture site infection in 1 patient and internal fixation breakage in 2 patients. 
Table I. Comparison of general clinical data between the DHS group and the PFNA group.

\begin{tabular}{|c|c|c|c|c|c|c|c|c|}
\hline \multirow[b]{2}{*}{ Group } & \multirow[b]{2}{*}{$\mathrm{N}$} & \multirow[b]{2}{*}{ Age (years) } & \multirow[b]{2}{*}{ Males/females (n) } & \multicolumn{4}{|c|}{ Evans type (n) } & \multirow[b]{2}{*}{ Complications n (\%) } \\
\hline & & & & Ia & $\mathrm{Ib}$ & Ic & Id & \\
\hline DHS & 50 & $70.3 \pm 6.2$ & $22 / 28$ & 15 & 14 & 16 & 5 & $29(58)$ \\
\hline PFNA & 50 & $68.2 \pm 7.4$ & $23 / 27$ & 13 & 16 & 14 & 7 & $33(66)$ \\
\hline$\chi^{2} / t$ & & 1.283 & 0.506 & \multicolumn{4}{|c|}{0.051} & 0.077 \\
\hline $\mathrm{P}$-value & & 0.315 & 0.477 & \multicolumn{4}{|c|}{1.256} & 0.943 \\
\hline
\end{tabular}

DHS, dynamic hip screw; PFNA, proximal femoral nail antirotation. Age is expressed as the mean \pm standard deviation.

Table II. Comparison of operative details between the DHS group and the PFNA group.

\begin{tabular}{lccccc}
\hline Group & $\mathrm{N}$ & $\begin{array}{c}\text { Operation time } \\
(\mathrm{min})\end{array}$ & $\begin{array}{c}\text { Length of hospital } \\
\text { stay (days) }\end{array}$ & $\begin{array}{c}\text { Intra-operative bleeding } \\
\text { volume (ml) }\end{array}$ & $\begin{array}{c}\text { Post-operative weight-bearing } \\
\text { time (days) }\end{array}$ \\
\hline DHS & 50 & $112.21 \pm 22.42$ & $24.54 \pm 4.98$ & $290.8 \pm 88.1$ & $12.32 \pm 3.47$ \\
PFNA & 50 & $78.45 \pm 24.38$ & $20.22 \pm 4.12$ & $163.3 \pm 78.3$ & $9.21 \pm 3.02$ \\
$t$ & & 5.484 & 3.015 & 0.513 & 2.318 \\
P-value & & 0.002 & 0.006 & 0.041 & 0.016 \\
\hline
\end{tabular}

DHS, dynamic hip screw; PFNA, proximal femoral nail antirotation.

Table III. Callusing time and swelling reduction time in the DHS group and the PFNA group.

\begin{tabular}{lccc}
\hline Group & $\mathrm{N}$ & $\begin{array}{c}\text { Callusing } \\
\text { time (days) }\end{array}$ & $\begin{array}{c}\text { Swelling reduction } \\
\text { time (days) }\end{array}$ \\
\hline DHS & 50 & $43.3 \pm 3.5$ & $13.6 \pm 5.0$ \\
PFNA & 50 & $31.5 \pm 3.1^{\text {a }}$ & $8.3 \pm 4.9^{\mathrm{a}}$ \\
\hline
\end{tabular}

${ }^{\mathrm{a}} \mathrm{P}<0.05$, PFNA vs. DHS. DHS, dynamic hip screw; PFNA, proximal femoral nail antirotation.

In the PFNA fixation group, 3 patients had complications (incidence rate, $6 \%$ ), of which 1 patient had hip varus, 1 patient had fracture site infection and 1 patient had internal fixation breakage. In comparison with the DHS group, the PFNA group had obviously fewer complications $(\mathrm{P}<0.05$; Table VI).

\section{Discussion}

Elderly patients with osteoporotic femoral intertrochanteric fracture should be treated with surgical therapy as soon as possible to restore fracture sites and recover hip motions at the early stage if the patient's constitution allows for surgical therapy. Reduction and internal fixation is routinely adopted for treatment, as the femoral intertrochanteric fracture site is cancellous bone, where blood supply is good. The most commonly used surgical methods are DHS and PFNA fixation.

DHS has the function of a tension band and achieves an ideal fixation effect through two types of pressure, i.e., static pressure and dynamic pressure (7). During internal fixation of DHS, hip screws anchor less sclerotin and have a poor antirotation effect, resulting in prolonged operation time and increased intra-operative bleeding volume. Poor osteoarticular blood supply affects late recovery, which is likely to delay the healing process $(8,9)$. Therefore, DHS fixation is not applicable in patients with severe osteoporosis or comminuted fracture in the lateral cortical bone of the femoral trochanter (10). Developed and designed on the basis of a PFN, PFNA is a novel intramedullary fixation system with an antirotation effect combined with a strong bearing (11). A series of biomechanical experiments (12-15) have confirmed that PFNA, due to its uniquely designed screw blade, has a high clinical application value because of the following points: i) It increases the contact area with the femoral bone and enhances the anchoring force of the blade. The blade is 'hammered' into the cancellous bone and may be automatically locked, which does not cause any loosening of the screw and avoids femoral shaft fracture caused by excessive local stress; ii) it has a good anti-cutout ability, anti-rotation and pressure effects, and biomechanics features in comparison with the PFN screw, which is conducive to applying close reduction and performing early rehabilitation exercise; iii) it has a precise positioning device, which requires opening of the lateral cortex only (without the requirement to remove the sclerotin), and thus, the integrity of the femoral trochanteric lateral cortex is retained. Due to all of these advantages, PFNA is widely used in patients with unstable comminuted intertrochanteric fracture or in frail osteoporosis patients (12).

Growth factors have important roles in the fracture healing process (16). TGF- $\beta 2$, an important growth factor, 
Table IV. Comparison of post-surgical TGF- $\beta 2$ expression between DHS group and PFNA group.

\begin{tabular}{lccccc}
\hline & & \multicolumn{3}{c}{ TGF- $\beta 2(\mu \mathrm{g} / \mathrm{l})$} \\
\cline { 3 - 6 } Group & $\mathrm{N}$ & 1 day & 7 days & 15 days & 30 days \\
\hline DHS & 50 & $217.4 \pm 25.6$ & $356.3 \pm 35.8$ & $408.7 \pm 35.6$ & $201.5 \pm 28.8$ \\
PFNA & 50 & $238.7 \pm 33.3$ & $390.4 \pm 40.6$ & $458.3 \pm 44.5$ & $223.4 \pm 31.5$ \\
$t$ & & 3.858 & 4.720 & 3.899 & 0.007 \\
P-value & 0.006 & 0.003 & 0.031 \\
\hline
\end{tabular}

DHS, dynamic hip screw; PFNA, proximal femoral nail antirotation; TGF, transforming growth factor.

Table V. Comparison of hip function scores between the DHS group and the PFNA group.

\begin{tabular}{|c|c|c|c|c|c|c|}
\hline \multirow[b]{2}{*}{ Group } & \multirow[b]{2}{*}{$\mathrm{N}$} & \multicolumn{3}{|c|}{ Cases with hip function score (n) } & \multirow[b]{2}{*}{ Poor } & \multirow[b]{2}{*}{ Excellent and good rate, $\mathrm{n}(\%)$} \\
\hline & & Excellent & Good & Fair & & \\
\hline DHS & 50 & 38 & 6 & 3 & 3 & $37(74.0)$ \\
\hline PFNA & 50 & 45 & 2 & 2 & 1 & $45(90.0)$ \\
\hline$\chi^{2}$ & & & & & & 2.76 \\
\hline P-value & & & & & & $<0.05$ \\
\hline
\end{tabular}

DHS, dynamic hip screw; PFNA, proximal femoral nail antirotation.

Table VI. Comparison of common complications between the DHS group and the PFNA group (n).

\begin{tabular}{|c|c|c|c|c|c|c|}
\hline Group & $\begin{array}{l}\text { Hip varus } \\
\text { n }[\operatorname{rate}(\%)]\end{array}$ & $\begin{array}{c}\text { Femoral shaft } \\
\text { fracture } \\
\mathrm{n}[\operatorname{rate}(\%)]\end{array}$ & $\begin{array}{c}\text { Cutout of femoral } \\
\text { head } \\
\mathrm{n}[\operatorname{rate}(\%)]\end{array}$ & $\begin{array}{c}\text { Fracture site } \\
\text { infection } \\
\mathrm{n}[\operatorname{rate}(\%)]\end{array}$ & $\begin{array}{c}\text { Internal fixation } \\
\text { breakage } \\
\mathrm{n}[\operatorname{rate}(\%)]\end{array}$ & $\begin{array}{c}\text { Total } \\
\text { incidence } \\
\text { rate }(\%)\end{array}$ \\
\hline DHS $(n=50)$ & $2(4)$ & $2(4)$ & $1(2)$ & $1(2)$ & $2(4)$ & 16 \\
\hline PFNA $(n=50)$ & $1(2)$ & $0(0)$ & $0(0)$ & $1(2)$ & $1(2)$ & 6 \\
\hline$\chi^{2}$ & & & & & & 3.98 \\
\hline P-value & & & & & & $<0.05$ \\
\hline
\end{tabular}

DHS, dynamic hip screw; PFNA, proximal femoral nail antirotation.

regulates the proliferation and differentiation of various cell types, including mesenchymal cells, osteoblasts, osteoclasts and chondrocytes, in the skeleton, increases the extracellular bone matrix, regulates the formation of bone and cartilage, promotes callus calcification and enhances bone repair. In different stages of fracture healing, the expression levels of TGF- $\beta 2$ are altered due to the impact of factors including the status of cell differentiation and growth conditions. It has been proved that TGF- $\beta 2$ stimulates collagen synthesis and is a strong chemokine. It stimulates mesenchymal cells to differentiate into chondrocytes during fracture healing. The net effect of this is to increase extracellular matrix and exert an important role in regulating bone and cartilage formation. At the same time, TGF- $\beta 2$ also promotes bone repair by stimulating the proliferation of osteoblasts and calcification of the callus. During the remodeling of the callus, the locally released TGF- $\beta 2$ coordinates the process of bone absorption and bone formation. TGF expression is dynamically expressed at different times of fracture healing. The expression levels of TGF gene are different at various stages of fracture healing, and are an important indicator in the evaluation of fracture prognosis; according to a previous study, the mRNA expression of TGF- $\beta 2$ reached a peak at 13 days after fracture (17).

The present study compared the peri-operative situation between patients receiving DHS and PFNA, indicating that operation time, length of hospital stay and intra-operative bleeding volume in the DHS group were greater than those in the PFNA group, and that the time to post-operative weight bearing was relatively long. Comparison of the recovery process between the two groups revealed that the callusing time and swelling reduction time in the PFNA vs. DHS group 
were $31.5 \pm 3.1$ vs. $43.3 \pm 3.5$ days and $8.3 \pm 4.9$ vs. $13.6 \pm 5.0$ days, respectively, and differences were statistically significant $(\mathrm{P}<0.05)$, indicating that the PFNA group had a faster post-operative fracture healing process. The results of the dynamic TGF- $\beta 2$ detection indicated that 7 days post-surgery, TGF- $\beta 2$ was increased compared with that at 1 day and reached a peak at 15 days, which is consistent with the results of a previous study (17). With the gradual healing of the fracture, the TGF- $\beta 2$ content had declined again at 30 days. The content of TGF- $\beta 2$ at $1,7,15$ and 30 days in the PFNA group was obviously higher than that at the identical time-points in the DHS group, indicating that PFNA stimulates the secretion of TGF- $\beta 2$ more than DHS and promotes the healing of the fracture. The possible reason is that PFNA internal fixation only requires a small incision and causes less trauma, with no need to perform open reduction and suturing, and has the nailing point at the tip of femoral trochanter. Therefore, it only causes mild damage to soft tissues of the fracture site, and at the same time, it only has a small impact on the femoral head, thereby promoting and increasing the secretion of TGF- $\beta 2$ in the body.

Hip function scores were compared at 3 months after the operation and the results indicated that in the DHS fixation group, the efficacy was rated as excellent in 38 patients, good in 6 patients, fair in 3 patients and poor in another 3 patients, with a rate of excellent and good efficacy of $74.0 \%$. In the PFNA fixation group, the efficacy was rated as excellent in 45 cases, good in 2 , fair in 2 and poor in 1 patient, with a rate of excellent and good efficacy of $90.0 \%$. Statistical analysis revealed that the rate of excellent and good efficacy in the DHS fixation group was lower than that in the PFNA group $(\mathrm{P}<0.05)$. In the DHS fixation group, complications occurred in 8 cases (incidence rate, 16\%), including 2 cases of hip varus, 2 cases of femoral shaft fracture, 1 case of cutout of the femoral head, 1 case of fracture site infection and 2 cases of internal fixation breakage; in the PFNA fixation group, complications were observed in 3 cases (incidence rate, 6\%), including 1 case of hip varus, 1 case of fracture site infection and 1 case of internal fixation breakage. In comparison with the DHS group, the PFNA group had obviously fewer complications $(\mathrm{P}<0.05)$. DHS surgery requires a relatively large incision and internal fixation is unstable. PFNA surgery only requires a small incision and the operation is relatively simple, without any peeling of deep soft tissue and periosteal reduction of the fracture site, and therefore, it has less impact on the local blood supply of the fracture, so that the blood supply of the fracture block is retained the most. Therefore, PFNA has a shorter operation time and lower bleeding volume, as well as a shortened fracture healing time in comparison with those in the DHS group (18).

In conclusion, PFNA fixation treatment, with its shorter operation time, lower intraoperative bleeding volume, less trauma, relatively faster fracture healing process, fewer complications and better curative effect, is more suitable for osteoporotic intertrochanteric fracture in elderly patients. As to the treatment of femoral intertrochanteric fracture, PFNA fixation is better than DHS fixation, which may be due to its high level of TGF- $\beta 2$ expression in comparison with that in the DHS group. The specific underlying mechanisms remains to be elucidated in further studies.

\section{Acknowledgements}

Not applicable.

\section{Funding}

This study was supported by the Research Project of the Jiangsu Health and Family Planning Commission (grant no. H201662).

\section{Availability of data and materials}

All data generated or analyzed during this study are included in this published article.

\section{Authors' contributions}

RX designed the present study; JR and YJ collected the data, FJ and DS analysed the data; JL and ZW interpreted the data; RX and DS prepared the manuscript. All authors read and approved the final manuscript.

\section{Ethical approval and consent to participate}

The present study was approved by the Ethics Committee of The Affiliated Hospital of Yangzhou University. Signed written informed consents were obtained from the patients and/or guardians.

\section{Consent for publication}

Not applicable.

\section{Competing interests}

The authors declare no competing interests.

\section{References}

1. Guo Y, Yang HP, Dou QJ, He XB and Yang XF: Efficacy of femoral nail anti-rotation of helical blade in unstable intertrochanteric fracture. Eur Rev Med Pharmacol Sci 21 (Suppl 3): 6-11, 2017.

2. Berger-Groch J, Rupprecht M, Schoepper S, Schroeder M, Rueger JM and Hoffmann M: Five-Year outcome analysis of intertrochanteric femur fractures: A prospective randomized trial comparing a 2-screw and a single-screw cephalomedullary nail. J Orthop Trauma 30: 483-488, 2016.

3. Gormeli G, Korkmaz MF, Gormeli CA, Adanas C, Karatas T and Simsek SA: Comparison of femur intertrochanteric fracture fixation with hemiarthroplasty and proximal femoral nail systems. Ulus Travma Acil Cerrahi Derg 21: 503-508, 2015.

4. Xu YZ, Geng DC, Mao HQ, Zhu XS and Yang HL: A comparison of the proximal femoral nail antirotation device and dynamic hip screw in the treatment of unstable pertrochanteric fracture. J Int Med Res 38: 1266-1275, 2010.

5. Carrington JL, Roberts AB, Flanders KC, Roche NS and Reddi AH: Accumulation, localization, and compartmentation of transforming growth factor beta during endochondral bone development. J Cell Biol 107: 1969-1975, 1988.

6. Strauss E, Frank J, Lee J, Kummer FJ and Tejwani N: Helical blade versus sliding hip screw for treatment of unstable intertrochanteric hip fractures: A biomechanical evaluation. Injury 37: 984-989, 2006.

7. Mereddy P, Kamath S, Ramakrishnan M, Malik H and Donnachie N: The AO/ASIF proximal femoral nail antirotation (PFNA): A new design for the treatment of unstable proximal femoral fractures. Injury 40: 428-432, 2009. 
8. Hong JY, Suh SW, Park JH, Shin YS, Yoon JR and Yang JH: Comparison of soft-tissue serum markers in stable intertrochanteric fracture: Dynamic hip screw versus proximal femoral nail-a preliminary study. Injury 42: 204-208, 2011.

9. Huang SG, Chen B, Zhang Y, Nie FF, Ju L, Li M and Zhang YH: Comparison of the clinical effectiveness of PFNA, PFLCP, and DHS in treatment of unstable intertrochanteric femoral fracture. Am J Ther 24: e659-e666, 2017.

10. Kumar R, Singh RN and Singh BN: Comparative prospective study of proximal femoral nail and dynamic hip screw in treatment of intertrochanteric fracture femur. J Clin Orthop Trauma 3: 28-36, 2012.

11. Scola A, Gebhard F, Dehner C and Roderer G: The PFNA(R) augmented in revision surgery of proximal femur fractures. Open Orthop J 8: 232-236, 2014

12. Li M, Wu L, Liu Y and Wang C: Clinical evaluation of the Asian proximal femur intramedullary nail antirotation system (PFNA-II) for treatment of intertrochanteric fractures. J Orthop Surg Res 9: 112, 2014.

13. Whatling GM and Nokes LD: Literature review of current techniques for the insertion of distal screws into intramedullary locking nails. Injury 37: 109-119, 2006.
14. Al-yassari G, Langstaff RJ, Jones JW and Al-Lami M: The AO/ASIF proximal femoral nail (PFN) for the treatment of unstable trochanteric femoral fracture. Injury 33: 395-399, 2002.

15. Lenich A, Mayr E, Ruter A, Mockl C and Fuchtmeier B: First results with the trochanter fixation nail (TFN): A report on 120 cases. Arch Orthop Trauma Surg 126: 706-712, 2006.

16. Centrella M, McCarthy TL and Canalis E: Transforming growth factor beta is a bifunctional regulator of replication and collagen synthesis in osteoblast-enriched cell cultures from fetal rat bone. J Biol Chem 262: 2869-2874, 1987.

17. Joyce ME, Jingushi S and Bolander ME: Transforming growth factor-beta in the regulation of fracture repair. Orthop Clin North Am 21: 199-209, 1990.

18. Helin M, Pelissier A, Boyer P, Delory T, Estellat C and Massin P: Does the PFNA nail limit impaction in unstable intertrochanteric femoral fracture? A 115 case-control series. Orthop Traumatol Surg Res 101: 45-49, 2015. 